Mitteilungen der Österreichischen Geographischen Gesellschaft,

156. Jg. (Jahresband), Wien 2014, S. 249-272

\title{
Neue Zuwanderung in die Alpen - Der Osten ist anders ${ }^{1)}$
}

\author{
Peter ČEde (Graz), Michael Beismann, Judith WALDER, Roland LöFfleR \\ und Ernst STEINICKE (alle Innsbruck)*
}

mit 7 Abb. und 1 Tab. im Text

\section{INHALT}

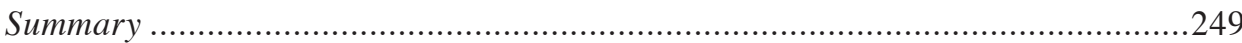

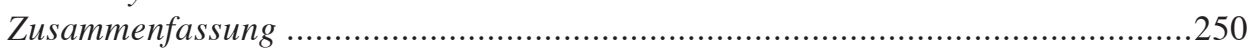

1 Problemstellung, Forschungsstand und Methodik ......................................251

2 New Highlanders in den Alpen - nur ein Phänomen im Westen? ...................253

3 Neuzuwanderer in Nordfriaul und im alpinen Westen Sloweniens -

Verbreitung und Potenzial ..................................................................25

4 Die demographischen Problemräume im alpinen Osten Österreichs ...............261

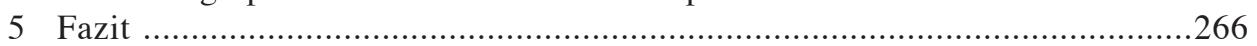

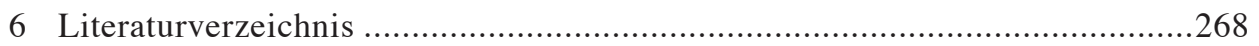

\section{Summary}

New immigration in the Alps - The East is different

The study focuses on the new immigration in the Alpine region, giving particular consideration to the previously insignificant amenity migration east of the European region Tyrol [Tirol]-South Tyrol [Südtirol/Alto Adige] - Trentino. Originating from the French Western Alps, the new immigration, after spreading first into the western Italian Alps, now also encompasses their eastern part (Montagna friulana) and, furthermore, - albeit with some retardation - the adjacent section of the Julian Alps [Julijske Alpe] (middle Isonzo [Soča] Region) in Slovenia. As a result of occupational

\footnotetext{
1) Die Studie wurde vom FWF (P25315-G16) unterstützt.

* ao.Univ.-Prof. Dr. Peter ČEDE, Institut für Geographie und Raumforschung, Universität Graz, A-8010 Graz; E-Mail: peter.cede@uni-graz.at,http://geographie.uni-graz.at; ao.Univ.-Prof. Dr. Ernst STEINICKE, Institut für Geographie, Universität Innsbruck, A-6020 Innsbruck, Innrain 52; E-Mail: ernst.steinicke@ uibk.ac.at, http://www.uibk.ac.at/geographie/; Mag. Michael BeISMAnN, Mag. Judith WaLder und Mag. Roland LÖFFLER, FWF-Arbeitsgruppe "Demographic Change in the Alps" (DCA-Forschungsgruppe), Institut für Geographie, Universität Innsbruck, A-6020 Innsbruck, Innrain 52; gemeinsames E-Mail: italian.alps@uibk.ac.at,http://www.uibk.ac.at/geographie/migration/
} 
and qualification-related relocation, the eastern Italian and Slovenian study areas experience a juxtaposition of simultaneous in-migration and out-migration patterns, characterised by a currently prevailing negative migration balance and declining population numbers. The persistent absence of amenity migration in the Alpine peripheral areas of eastern Austria, combined with continuing population losses, have now effectively turned this region into the largest demographic problem area in the Alps. An analysis of the causes of the insignificant immigration volume in the eastern part of the Austrian Alps reveals several factors that are interrelated to varying degrees, such as low scenic attractiveness, development deficits, forestry, large-scale landholding, and an overall negative image. These influences are responsible for the fact that in Alpine eastern Austria there is no potential for amenity migration on a larger scale. Therefore, in this region, the turnaround in the demographic trend cannot be attributed to a temporal delay. Thus, the new immigration, particularly in the crystalline mountain ranges of Carinthia [Kärnten] and Styria [Steiermark] and the Styrian and Lower Austrian Limestone Alps [Steirische und Niederösterreichische Kalkalpen], will continue to remain sparse in the future.

\section{Zusammenfassung}

Die Studie wirft einen Blick auf die neue Zuwanderung im Alpenraum unter besonderer Berücksichtigung der bislang unbedeutenden Amenity Migration östlich der Europaregion Tirol - Südtirol - Trentino. Ausgehend von den französischen Westalpen erfasst diese - nach den italienischen Westalpen - mittlerweile auch den östlichen Teil der italienischen Alpen (Montagna friulana) sowie mit zeitlicher Verzögerung zudem den angrenzenden Abschnitt der Julischen Alpen [Julijske Alpe] in Slowenien (mittleres Isonzo- [Soča-]gebiet). Dabei ergibt sich in den ostitalienischen und slowenischen Untersuchungsgebieten aufgrund von berufs- und qualifikationsbedingten Abwanderungen zeitgleich ein Nebeneinander von Weg- und Zuzügen bei einem gegenwärtig noch leicht dominierenden negativen Migrationssaldo und rückläufigen Einwohnerzahlen. Die bis dato nicht vorhandene Amenity Migration in den alpinen Peripherräumen Ostösterreichs, gepaart mit den weiterhin anhaltenden Bevölkerungsverlusten, machen diese Region zum mittlerweile größten demographischen Problemgebiet der Alpen. Eine Analyse der Ursachen dafür ergibt im östlichen Teil der österreichischen Alpen mehrere, unterschiedlich stark miteinander verknüpfte Faktoren (geringe landschaftliche Attraktivität, Erschließungsdefizite, Forstwirtschaft, Großgrundbesitz, Negativimage), die dafür verantwortlich sind, dass dem alpinen Osten Österreichs weitgehend das Potenzial für Amenity Migration in größerem Umfang fehlt. Daher ist hier auch nicht eine zeitliche Verzögerung in der demographischen Trendwende anzunehmen. Dadurch wird die neue Zuwanderung insbesondere in den kristallinen Mittelgebirgen Kärntens und der Steiermark sowie in den Steirisch-Niederösterreichischen Kalkalpen auch in Zukunft weiterhin gering bleiben. 


\section{Problemstellung, Forschungsstand und Methodik}

Zum Themenkreis Hochgebirge als neuer Zuwanderungsraum liegt sowohl im Hinblick auf verschiedene Gebirge als auch hinsichtlich theoretischer Überlegungen mittlerweile ein umfangreiches wissenschaftliches Schrifttum vor (STEWART 2002; Moss 2006; Moss et al. 2009; Löffler \& Steinicke 2007; MCIntyre 2009; Gosnell \& ABrams 2011 oder Abrams et al. 2012). Seit knapp zehn Jahren diskutieren Geographen und Bevölkerungsforscher dieses Phänomen auch in Bezug auf die Alpen, wobei der Amenity Migration und den neuen Formen des multilokalen Wohnens - besonders was die abgelegenen Gebirgsregionen betrifft - eine besondere Rolle zugeschrieben werden (Perlik 2006, 2008; Pascolini 2008; Corrado 2010; Messerli et al. 2011; Perlik 2011; Beismann et al. 2012; Bender \& Kanitscheider 2012, 2013; Corrado et al. 2014).

Der vorliegende Beitrag baut auf einer Reihe von Fallstudien in demographischen Problemgebieten in den italienischen Alpen (zuletzt Beismann et al. 2012; Steinicke et al. 2011, 2012, 2013, 2014) auf, in denen eindeutig belegt werden konnte, dass in verschiedenen Gebieten die jahrzehntelang vorherrschende Abwanderung durch eine neue Zuwanderung abgelöst wurde. So lässt sich aus den amtlichen Statistiken seit dem Jahr 2001 eine Nettozuwanderung in peripheren Berggemeinden (vgl. Di GiorA 2013) von knapp 40.000 Personen (das sind über 3.000 Personen pro Jahr) ermitteln (SteinicKe et al.2012, S. 334). Im Zuge dessen wurden Modelle entwickelt, welche die nunmehr veränderte Zusammensetzung der Bevölkerung in peripheren Alpensiedlungen darstellen: von ganzjährig ansässigen Locals über Touristen, Saisonarbeiter, Inhaber von Zweit-/Ferienwohnsitzen bzw. multilokal-wohnenden Amenity-Migranten bis hin zu counterurbanen Newcomers.

Die New Highlanders sind Personen, die sich bewusst für ein Leben in den Bergen entscheiden, die außeralpine Städte (für längere Perioden) verlassen und zu einer Revitalisierung und somit zum Erhalt von peripheren Gebirgssiedlungen beitragen. Bezüglich Motiven und Herkunftsgebieten von Amenity-Migranten sei hier auf die Arbeiten von STEINICKE et al. (2012) und WALDER et al. (2008) verwiesen, in denen ausführlich auf diese Thematik eingegangen wird. Ausgehend vom Innovationsraum der französischen Alpen der 1960/70er Jahre diffundierten diese Prozesse in die italienischen Westalpen mit den Ballungsräumen der Poebene [Pianura Padana, Padania] als erheblichem Potenzial für den Zustrom urbaner Bevölkerung in das Gebirge und erfassen nunmehr auch die italienischen Ostalpen und ebenfalls das mittlere slowenische Isonzo-[Soča] Gebiet. Die derzeitigen Bevölkerungsverluste stehen dazu nicht im Widerspruch, zumal die Zuwanderungsgewinne oft durch die äußerst ungünstigen bevölkerungsbiologischen Verhältnisse kompensiert werden - ein Resultat der Emigrationsperiode. Da auch Untersuchungen in der Schweiz das Phänomen der neuen Zuwanderung bestätigen (Schuler et al. 2004; Hornung \& RöThlisberger 2005; CAMEnisch \& DebarBIEUx 2011; Messerli et al. 2011), lässt der bisherige Forschungsstand insgesamt die Aussage zu, dass diese demographische Entwicklung von Westen nach Osten vorrückt. 
Ziel dieses Beitrags ist es zu untersuchen, inwieweit die demographischen Problemgebiete im alpinen Osten Österreichs sowie in den slowenischen Alpen von der neuen Zuwanderung betroffen sind oder ob es dort ein solches Phänomen überhaupt gibt. Sollte dies nicht der Fall sein, so stellt sich die Frage, ob „,fehlende“ Amenity landscapes (WALTERT \& SCHLÄPFER 2010) als Begründung dafür geltend zu machen sind.

Basis für die neuen Erkenntnisse ist eine gesamtalpine Analyse der Bevölkerungsentwicklung und des Wanderungsgeschehens auf kommunaler Ebene. In einem zweiten Schritt werden die aktuellen demographischen Problemgebiete der Ostalpen hinsichtlich Zielsetzung und Fragestellung näher untersucht, wobei die gegenwärtige Entwicklung im friaulischen Gebirge und in den südlichen slowenischen Alpen genauer dargelegt wird. Im Zentrum der Betrachtungen steht jedoch die überaus starke Entvölkerung im alpinen Osten Österreichs.

Die Studie stützt sich auf einen Methodenpool, der dadurch gekennzeichnet ist, unterschiedliche Zugänge zur Thematik miteinander zu vernetzen. Neben der Berücksichtigung des aktuellen Forschungsstandes wurden die amtlichen Ergebnisse der Volkszählungen aller Alpenstaaten seit der zweiten Hälfte des 20. Jhs. sowie die darauf Bezug nehmenden thematischen Karten der letzten Jahrzehnte systematisch ausgewertet. Zusätzlich zu diesen quantitativen Arbeitsgrundlagen lieferten mehrere Forschungsaufenthalte der Autoren in den Untersuchungsgebieten entscheidende Erkenntnisse. Zu den wichtigsten Arbeitsmethoden vor Ort zählten dabei - neben eigenem mehrjährigen Monitoring - teilstrukturierte Interviews sowohl mit Einheimischen als auch mit Neuzuwanderern, Workshops mit kommunalen Amtsleitern und Bürgermeistern sowie Gespräche mit Regionalmanagern der EU-kofinanzierten lokalen Arbeitsgruppen und Regionalverbände. Als Erhebungsmethode für quantitativ und qualitativ relevante Einblicke in den Prozess der Neu- und Wiederbesiedelung peripher liegender Siedlungen erwiesen sich zudem standardisierte Funktionskartierungen als zielführend.

Für die Montagna friulana sowie für die Julischen Alpen [Julijske Alpe] im italienisch-slowenischen Grenzraum liegen bereits erste Untersuchungsergebnisse vor, auf die sich die vorliegende Analyse sowohl theoretisch als auch empirisch stützen kann (STEINICKE et al. 2012). Während sich die Entwicklung im friaulischen Teil somit unter Bezugnahme auf bereits vorhandene eigene Forschungsergebnisse analysieren lässt, gehen die qualitativen Aussagen für den slowenischen Bereich auf Leitfadeninterviews zurück. Die Auswahl der Interviewpartner erfolgte im Schneeballverfahren, wobei die teilstrukturierte Befragungstechnik auch Gruppendiskussionen miteinbezog. In jedem der ausgewiesenen Teilräume kamen zwischen zehn und 20 Interviews zur Auswertung. Für das Isonzogebiet lagen aus früheren Studien zudem Ergebnisse von standardisierten Befragungen vor (STEINICKE et al. 2012, 2014). Im österreichischen Untersuchungsgebiet wurden in den jeweiligen Gemeindeämtern Fragebogenerhebungen über die aktuelle Bedeutung von Neuzuwanderern durchgeführt.

Das Untersuchungsgebiet - die Alpen - wurde nicht nach streng topographischgeomorphologischen Gesichtspunkten, sondern nach den Kriterien der Alpenkonvention (EURAC 2004) abgegrenzt. 


\section{New Highlanders in den Alpen - nur ein Phänomen im Westen?}

Die folgenden Ausführungen zur Bevölkerungsdynamik in den Alpen stützen sich auf die Analyse relevanter demographischer Kennziffern. Um das statistische Bild der derzeitigen Situation mit dem vorangegangener Jahrzehnte vergleichen zu können, wurden die Karten der Bevölkerungsentwicklung im Alpenraum von 1951 bis 1981 und von 1981 bis 2000 von BäTZING (2002) herangezogen, generalisiert und aktualisiert (vgl. Abb. 1). Aufgrund unterschiedlich langer Vergleichszeiträume waren die Schwellenwerte in Relation zu setzen. Alle drei Darstellungen zeigen die Gebiete mit signifikanten Einwohnerverlusten. Galten bis zu Beginn der 1980er Jahre hauptsächlich noch die westlichen und südlichen Alpenteile als die großen demographischen Problemgebiete der Alpen, so hat sich das Bild im Laufe der darauffolgenden

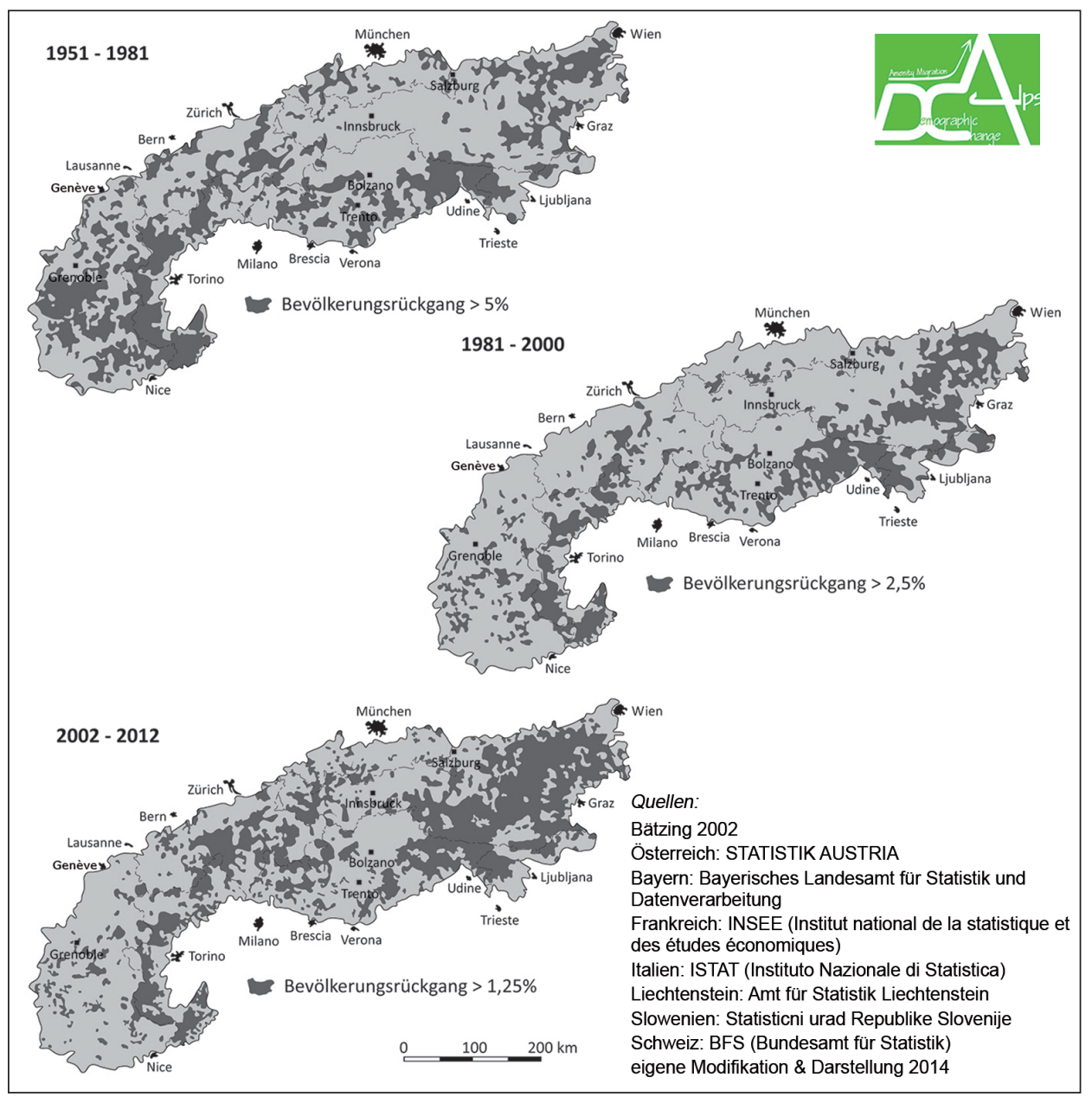

Abb. 1: Entwicklung demographischer Problemgebiete im Alpenbogen von 1951 bis 2012 


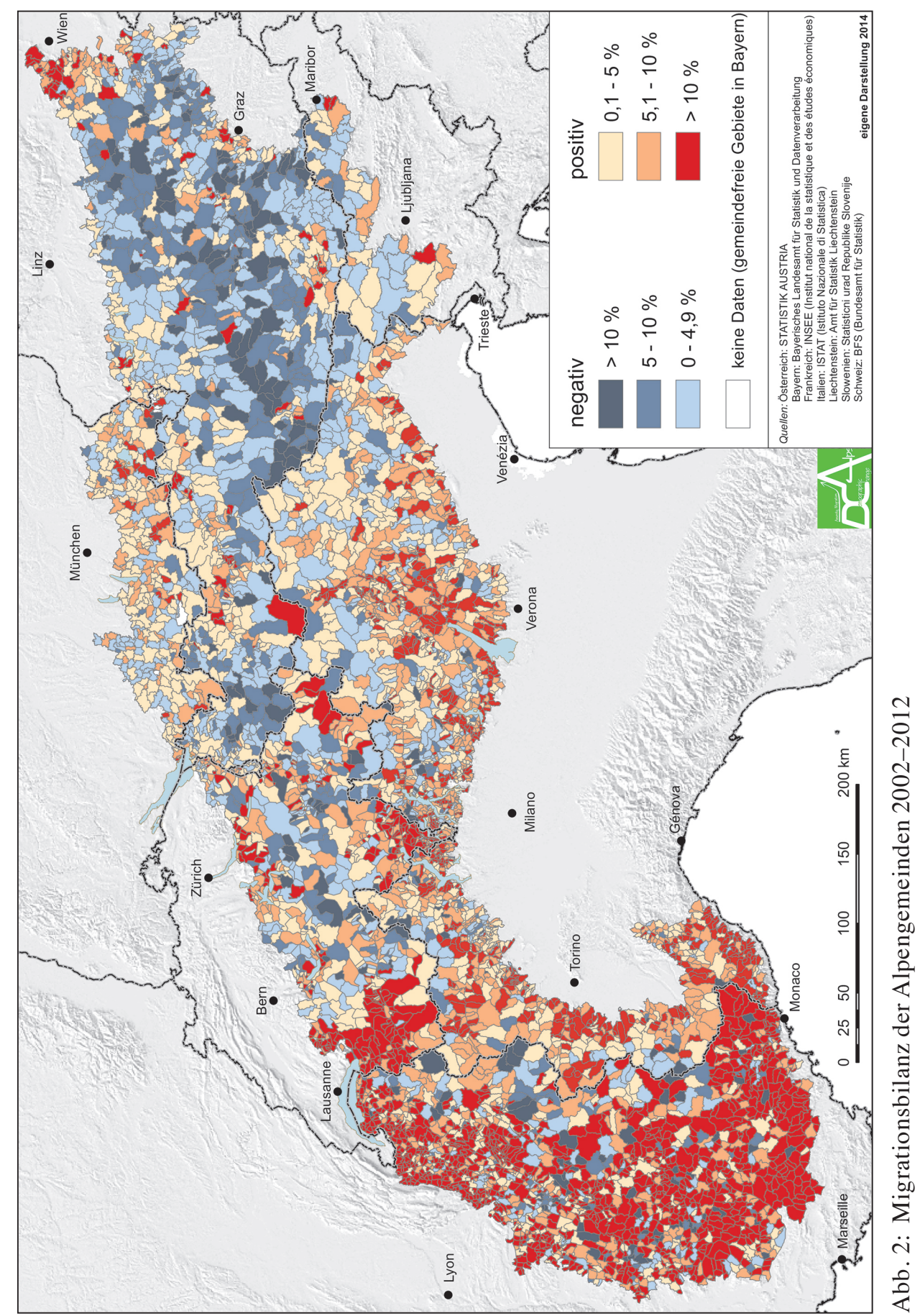


Jahrzehnte stark gewandelt: Während sich im Westen die Gemeinden nach und nach von der massiven Abwanderungsphase erholt haben, erscheint die Situation im Osten inzwischen prekär geworden zu sein. Speziell die Gebiete östlich der Europaregion Tirol-Südtirol-Trentino treten noch immer mit großen Einwohnerrückgängen hervor. Den Westalpen, wo sich die Problemgebiete immer mehr reduzieren und zu Zuwanderungsräumen werden, stehen somit beinahe flächendeckende Bevölkerungsverluste in Nordfriaul, den alpinen Teilen Westsloweniens, vor allem aber im östlichen Abschnitt der österreichischen Alpen gegenüber.

Noch deutlicher tritt der demographische West-Ost-Gegensatz zutage, wenn man sich auf die Darstellung der Wanderungsdynamik beschränkt. So zeigt Abbildung 2 die Netto-Zuwanderungsgebiete der Alpen, die nunmehr in erster Linie im Westen liegen. Aber auch die italienischen Alpen gehören zumindest bis zum Bellunese dazu.

Die italienischen Ostalpen sowie das alpine Westslowenien befinden sich gegenwärtig in einem demographischen Transformationsprozess. Zwar sind die Entvölkerungswerte immer noch beträchtlich, doch spielt bei der Bevölkerungsentwicklung mittlerweile das Geburtendefizit die Hauptrolle, was sich als biodemographische Konsequenz der jahrzehnte- bis jahrhundertelangen Auswanderung erklären lässt. Abwanderungen sind zweifellos noch demographisch wirksam, doch nimmt ihr Ausmaß stetig ab. Dies belegen die rückläufigen Entvölkerungsraten Nordfriauls (Montagna friulana) eindrucksvoll (vgl. Tab. 1).

\begin{tabular}{|c|c|c|c|}
\hline Periode & Entvölkerung p.a. & Periode & Entvölkerung p.a. \\
\hline $1952-1961$ & 1,05 & $1982-1991$ & 0,97 \\
\hline $1962-1971$ & 2,29 & $1992-2001$ & 0,72 \\
\hline $1972-1981$ & 1,09 & $2002-2011$ & 0,18 \\
\hline
\end{tabular}

Quelle: ISTAT; http://www.demo.istat.it/; eigene Berechnungen

Tab. 1: Abnahme der Entvölkerung in den friaulischen Alpen (in \%)

\section{Neuzuwanderer in Nordfriaul und im alpinen Westen Sloweniens - Verbreitung und Potenzial}

Wie die Abbildungen 1 und 2 deutlich zeigen, sind die Regionen im alpinen Osten Österreichs mittlerweile zum flächen- und zahlenmäßig größten Abwanderungsgebiet der Alpen geworden. Bevor darauf näher eingegangen wird, sollen zunächst die neuen Bergbewohner in der Montagna friulana thematisiert werden. Dieser Gebirgsteil, der seit dem Zweiten Weltkrieg alpenweit die größten Bevölkerungsverluste hinnehmen musste, eignet sich besonders, um die Effekte der aktuellen Zuwanderung modellhaft aufzuzeigen. Aufschlussreich ist des Weiteren ein Blick über die Grenze in den slowenischen Alpenraum, wo während der kommunistischen Periode die Abwanderung schwächer als im benachbarten Nordfriaul war. 


\subsection{Montagna friulana}

$\mathrm{Ab}$ dem Ende des Zweiten Weltkrieges waren die friaulischen Berggemeinden von einer massiven Entvölkerung gekennzeichnet, zusätzlich beschleunigt durch die Erdbebenserie des Jahres 1976, welche den Siedlungsraum weitflächig zerstörte. Auch heute zeigt der statistische Befund insgesamt Bevölkerungsverluste, sodass man keinesfalls annehmen würde, hier auf Spuren einer demographischen Trendwende zu stoßen. Im Rahmen von mehreren Forschungsaufenthalten konnte jedoch festgestellt werden, dass sich die neuesten demographischen Entwicklungen in diesem Raum nicht mehr von jenen in den italienischen Westalpentälern unterscheiden. Zwar gibt es Auswärtige noch immer in weitaus geringerer Zahl, doch trifft man auch in äußerst peripheren Dörfern auf neue Bewohner außeralpiner Herkunft. Dass die gesamte Entwicklung im Osten der italienischen Alpen langsamer verläuft, ist erstens, wie bereits erläutert, auf die ungünstigen biodemographischen Strukturen zurückzuführen, welche wiederum aus der vorhergegangenen Abwanderungsperiode resultieren. Zweitens fehlen hier im Unterschied zu den italienischen Westalpen - große Verdichtungsräume wie Turin [Torino], Mailand [Milano] oder Genua [Genova], die sowohl einen enormen Druck auf Umland und Exurbia ${ }^{2}$ ausüben als auch einen großen Pool für potenzielle AmenityMigranten darstellen. Zudem sei aber betont, dass es neben der neuen Zuwanderung weiterhin zu berufsorientierten Fortzügen kommt. Dadurch erfolgt mancherorts ein regelrechter Bevölkerungsaustausch, den PerLIK (2011, S. 8) als „Alpine Gentrification" bezeichnet.

Handelt es sich in vielen Fällen nicht unbedingt um ein Massenphänomen, so ist der Prozess der Amenity Migration in Friaul gegewärtig doch für eine Wiederbelebung peripherer Siedlungen verantwortlich. Auch wenn die Wiederbesiedelung in Personenzahlen gemessen nicht immer statistisch relevant ist, so sind oft schon einige wenige Zuwanderer in der Lage, für eine Neubelebung der Gemeinde zu sorgen.

Positive Auswirkungen der Newcomers in entlegenen Hochgebirgsregionen der Alpen und daher auch in der Montagna friulana sind die Stabilisierung oder Revitalisierung lokaler Strukturen und Gebäude sowie die damit verbundene Neubesiedelung von Ortswüstungen (vgl. Abb. 3). Zudem tragen viele Amenity-Migranten durch Innovationen und Unternehmergeist entscheidend zum Aufschwung der kommunalen Wirtschaft bei. Selbst jene, die nicht das ganze Jahr dort leben, leisten einen Beitrag durch den Erhalt ihrer Häuser, den die einheimische Bevölkerung allein nicht mehr bewerkstelligen kann. Die New Highlanders verhindern darüber hinaus eine (weitere) Überalterung und wirken auch rückläufigen Geburtenzahlen entgegen.

Während sich die Auswirkungen auf die soziodemographische Zusammensetzung der Bevölkerung erst langsam bemerkbar machen, lässt sich die Einflussnahme der Zuwanderer auf Tradition und Kultur rascher nachweisen. Untersuchungen in der deutschen Sprachinsel Zahre/Sauris in Westfriaul zeigten beispielsweise, dass die neuen Zuwanderer zwar der Bewahrung des lokalen Idioms keinesfalls förderlich sind,

\footnotetext{
2) Im Sinne von Wohngebieten außerhalb städtischer Vororte, die vorwiegend von wohlhabenden Pendlern bewohnt werden.
} 


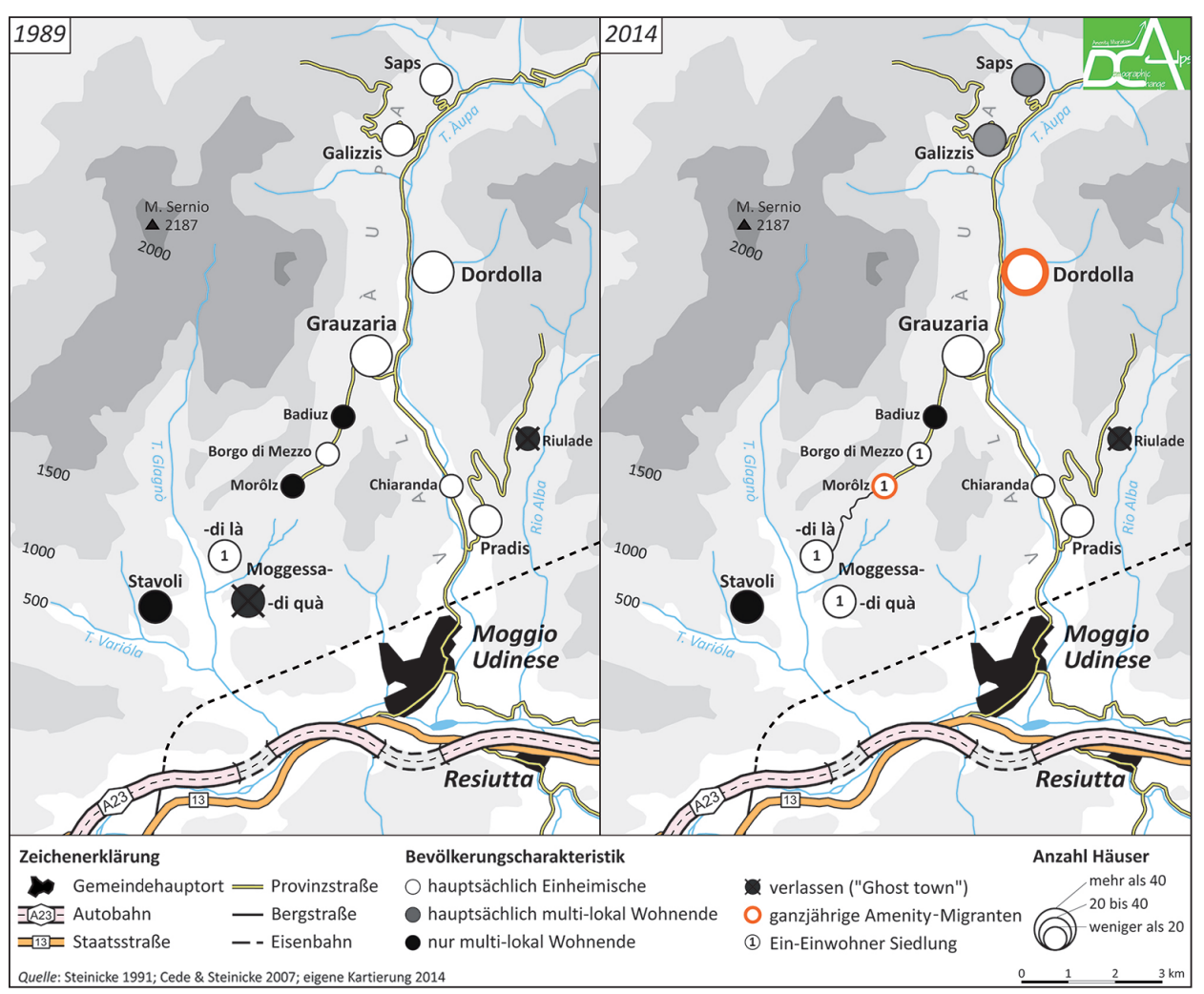

Abb. 3: Amenity-Migranten im Val Àupa 1989 und 2014

jedoch durch verschiedene Aktivitäten hervortreten, die regionale kulturelle Strukturen stärken. Ähnliches konnten die Autoren auch in anderen alpinen Minderheitenräumen feststellen (WALDER et al. 2008; STEINICKE et al. 2014).

\subsection{Im Süden des Triglav}

Während sich eine Fülle von Studien mit Freizeitwohnsitzen sowie ländlicher Siedlungsentwicklung und -struktur in den slowenischen Alpen befasst (z.B. Gosar \& Roblek 2001; Bufon 2003; Ravbar 2004; Gosar 2005, 2009), ist von slowenischer Seite bislang keine Arbeit bekannt geworden, welche Amenity Migration in den Mittelpunkt ihrer Betrachtung stellt. Zwar gehen LAMPIČ \& MRAK (2012) auf diese Thematik ein, jedoch untersuchen sie lediglich außeralpine Räume Sloweniens.

Um die Existenz und Verbreitung von Neuzuwanderern in abgelegenen demographischen Problemgebieten der slowenischen Alpen zu erforschen, bieten sich in den Julischen Alpen sechs zusammenhängende Großgemeinden [občine] an, die allesamt eine negative Wanderungsbilanz aufweisen und - außer im Westen, wo sie an Friaul, 


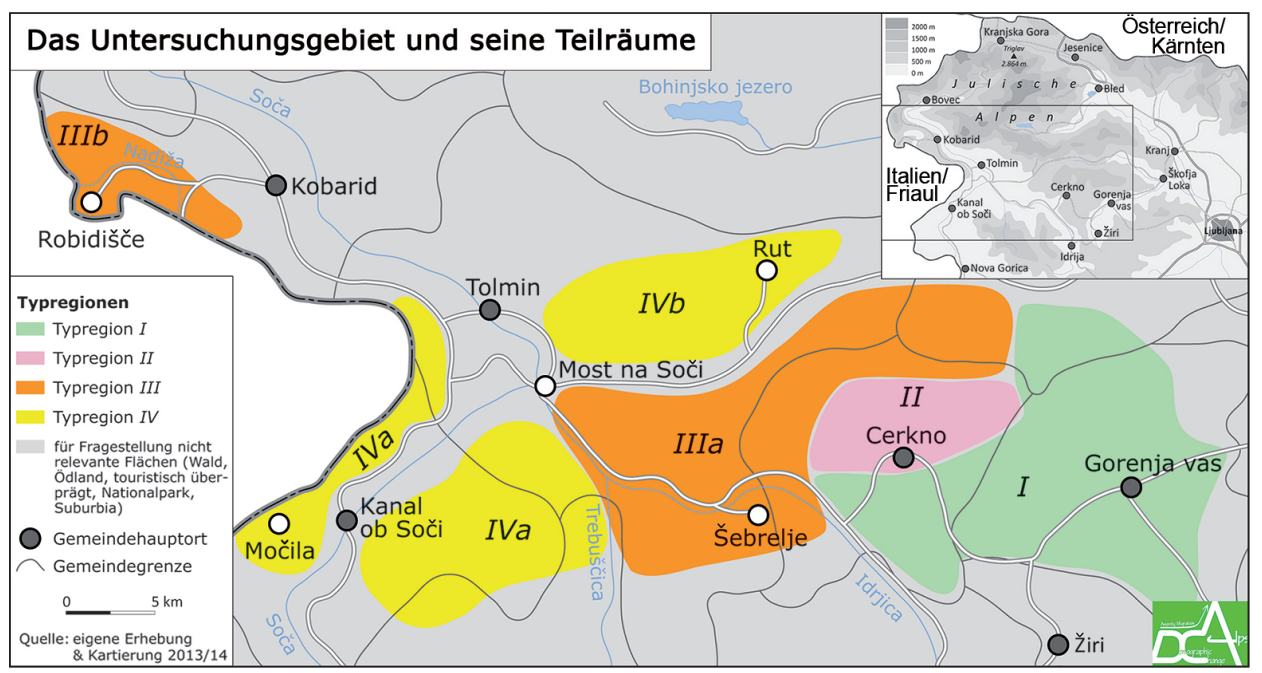

Abb. 4: Amenity Migration in den südlichen slowenischen Alpen

und im Osten, wo sie an urban geprägte Räume angrenzen - von ländlichen Zuwanderungsgemeinden umschlossen werden (vgl. Abb. 4).

Mögen sich die kommunistischen Mechanismen ex-Jugoslawiens im Alpengebiet strukturerhaltend ausgewirkt haben, so sind doch mittlerweile große Teile der traditionell (berg-)bäuerlich geprägten Bevölkerung in Siedlungen höherer Zentralität abgewandert. In den peripheren ländlichen Regionen abseits der Tourismuszentren ist deshalb nicht zu erwarten, dass eine Welle neuer Zuwanderer - auf der Suche nach höherer Lebensqualität - die fortgezogene lokale Bevölkerung zahlenmäßig ersetzt. Dazu fehlt es auch hier an nah gelegenen Ballungsräumen. Dennoch stellt sich - ähnlich wie in Nordfriaul - die Frage, ob es im Süden des Triglav zumindest einige Newcomers gibt, die zur Revitalisierung und zum Erhalt des ruralen Siedlungsraumes beitragen und möglicherweise den Beginn einer stärkeren Zuwanderung signalisieren.

In slowenischen Großgemeinden ist die Auswertung der offiziellen Wanderungsstatistik für die vorliegende Fragestellung nicht zielführend. Beispielsweise zählte der Ort Tolmein [Tolmin] im Jahr 2012 3.525 Einwohner (STATISTIČNi URAd Republike SLOVENIJE 2013), sein Gemeindegebiet hatte allerdings 11.628 Bewohner, die sich auf 72 Ortschaften [naselja] verteilten, die über 50 Straßenkilometer voneinander entfernt liegen. Um Konkretes über die Bevölkerungsdynamik auf Ortsteilebene erfahren zu können, sind qualitative Untersuchungen unerlässlich. In Anbetracht der zahlreichen Einzelsiedlungen und Weiler wäre es aber schwierig und unzureichend, einige Orte stichprobenartig zu untersuchen, weshalb eine intensive Bereisung des Untersuchungsgebietes (vgl. Abb. 4) durch das Forscherteam notwendig war. Auf Basis der Erfassung spezifischer Merkmale der Bau- und Siedlungsphysiognomie in den Ortsteilen wurden im Schneeballverfahren Interviews zur jeweiligen lokalen Situation geführt. Dement- 
sprechend entstand ein brauchbar enges Netz an Einzelinformationen, welche mittels GIS-Unterstützung verortet, verknüpft und visualisiert wurden. Dadurch ließ sich das Untersuchungsgebiet hinsichtlich der „Einflüsse von außen“ in Teilräume typisieren. Die Ergebnisse sind in Abbildung 4 zusammengefasst. Dabei wurden in den für die Fragestellung relevanten Räumen insgesamt vier Typen ausgeschieden, die Bestand und Potenzial an Amenity Migration widerspiegeln.

\section{Typregion I: Übergangszone zur Exurbia}

Dieser Regionstyp liegt noch in Tagespendler-Distanz zu den wirtschaftlichen Aktivräumen um Bischoflack [Škofja Loka], Krainburg [Kranj] und Laibach [Ljubljana] sowie zu den Siedlungen mit höherrangigen zentralörtlichen Funktionen in der Nähe der Schnellstraße/Autobahn Richtung Adria und Nova Gorica. Hier lassen sich zwar einige wenige Newcomers aus außeralpinen Regionen ausmachen, doch nehmen diese kaum Einfluss auf die bestehende Bausubstanz und den Erhalt der Kulturlandschaft, der zumindest mittelfristig endogen gesichert scheint.

\section{Typregion II: Peripherie mit touristischen Ansätzen}

Die Bewaldung dringt hier vor, Verbuschung zeugt von der Abnahme landwirtschaftlicher Nutzung, und im Vergleich zum Regionstyp I verweist die weniger gut instand gehaltene Bausubstanz auf Abwanderung. Demgegenüber lassen sich an landschaftlich attraktiven Standorten Neu- und Rohbauten erkennen, die mit touristischen Aktivitäten zusammenhängen, desgleichen vereinzelt Zweit-, Ferien- und Sommerwohnsitze. Dennoch bilden sowohl die Rückwanderer als auch die neuen Zuwanderer bei weitem kein Massenphänomen, sie unterstützen aber unter anderem das örtliche Baugewerbe. Das Schigebiet Cerkno erfreut sich steigender Beliebtheit, gewinnbringender Tourismus und in der Folge ein verstärktes Einströmen von Amenity-Migranten lassen allerdings noch auf sich warten.

\section{Typregion III: Amenity landscapes}

Diese Typregion, die um die untere Idrijca, nordöstlich davon (IIIa) sowie im äußersten Westen des Untersuchungsgebiets am Natisone [Nadiža] (IIIb) zu finden ist, bildet das in mehrfacher Hinsicht interessanteste Gebiet bezüglich des Einflusses von Newcomers auf die lokalen Gegebenheiten. Auf den ersten Blick würde man in der Typregion IIIa mit deren beinahe geschlossener Bewaldung und mit ihrer Abgeschiedenheit keine Amenities als Pullfaktoren einer Zuwanderung erwarten. Abseits der Hauptverkehrswege finden sich allerdings Rodungsinseln und landwirtschaftlich genutzte Hochflächen mit Dörfern in ansprechender Lage. Die Gebiete von Šebrelje (IIIa) und im Westen von Karfreit [Kobarid] (IIIb) überraschen mehrfach durch großzügig umgestaltete und frisch renovierte Bausubstanz, die zumeist nicht aus endogenen Ressourcen entstanden ist. 
Tatsächlich lassen sich hier zwei Varianten externer Einflüsse feststellen: Durch eine Billigfluglinie zwischen London und Marburg [Maribor] in den Jahren 2007 und 2008 und stark beworben von britischen Maklerbüros strömte zumindest ein Dutzend Briten in das Gebiet an der unteren Idrijca, kaufte dort Wohnraum und begann, diesen zu sanieren. Obwohl die Instandsetzung persistenter Bausubstanz mit der Einstellung der Direktverbindung wieder vorbei zu sein schien, waren die Weichen für eine individuelle neue Zuwanderung gestellt, die insbesondere die reizvolle Landschaft am Natisone erfasste (IIIb). Die neuen Bergbewohner kommen aus den größeren Städten im Süden Sloweniens, doch sind auch auffallend viele aus dem Ausland darunter. Ebenso unterschiedlich sind die Motive, die von billigem Wohnen mit hohem Freizeitwert über Zufall bis hin zum Wunsch, ungestört alternative Landwirtschaft betreiben zu können, reichen. Die meisten Newcomers werden - nicht zuletzt durch ihr innovatives Wirken - von den Locals positiv konnotiert.

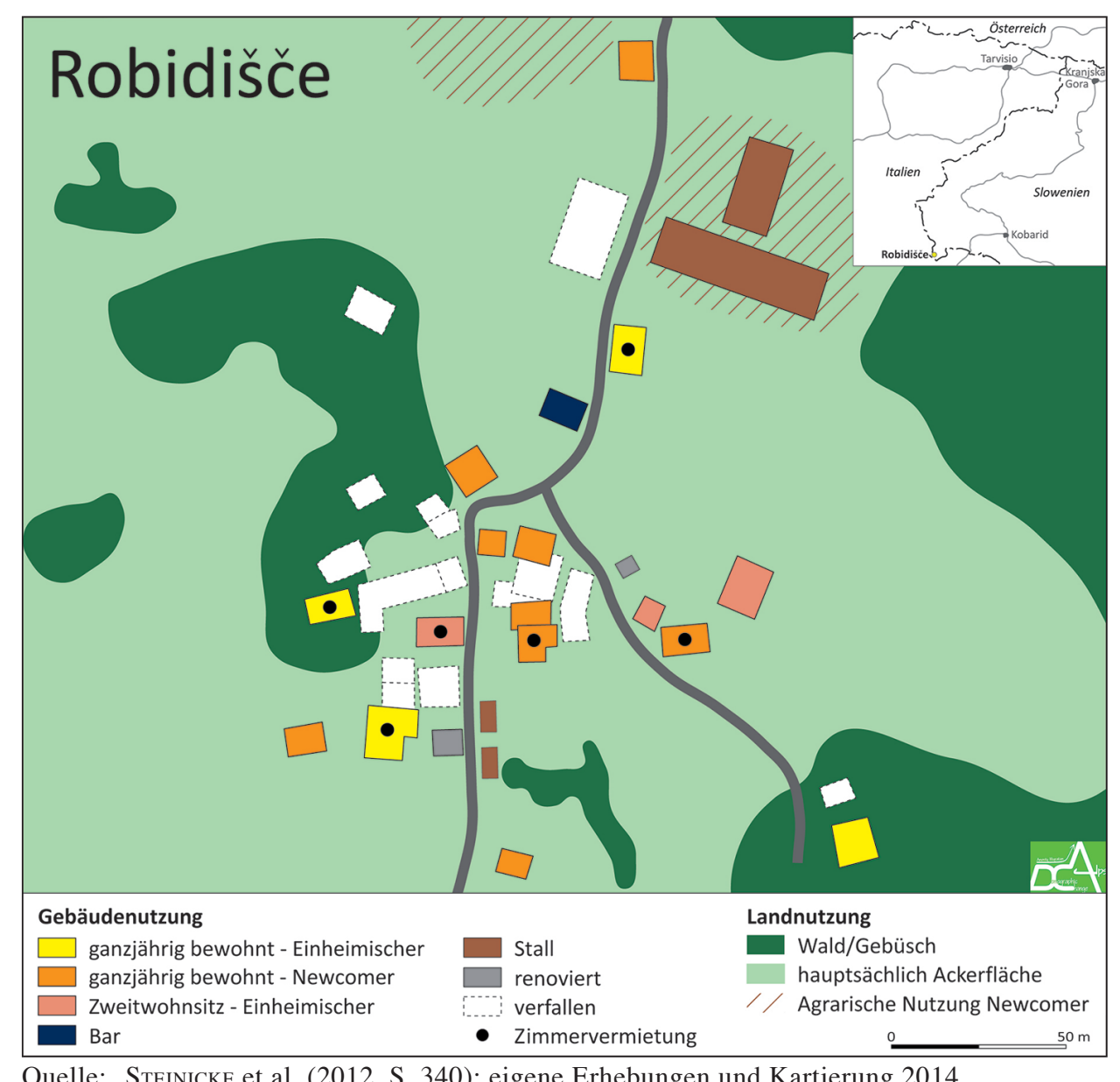

Quelle: Steinicke et al. (2012, S. 340); eigene Erhebungen und Kartierung 2014

Abb. 5: Gebäude- und Flurnutzung in Robidišče (Gemeinde Karfreit [Kobarid]) 2014 
Das Beispiel des Dorfes Robidišče (vgl. Abb. 5), das unmittelbar nach dem Zweiten Weltkrieg über 100 Einwohner zählte und zu Anfang der 2000er Jahre nur noch sieben permanente Bewohner hatte, stellt den Modellfall einer Amenity-Siedlung dar (Steinicke et al. 2012, S. 340). Im Sommer 2014 lebten hier bereits wieder 36 Personen (ohne Touristen). In den letzten beiden Jahren ist der Wohnraum stark ausgebaut worden und er steht zur Vermietung an Touristen, aber auch für Gastarbeiter, die im nahen Italien einer Beschäftigung nachgehen, bereit.

\section{Typregion IV: Ungenutztes Potenzial für Amenity Migration}

Das im Westen der Typregion IIIa dies- und jenseits des Isonzo liegende Gebiet (IVa), unterscheidet sich naturräumlich von ersterer nur unwesentlich. Die ansprechende Landschaft sollte eigentlich Newcomers anziehen, doch hat sie die Amenity Migration noch nicht erfasst. Die Ortskerne zeigen deutliche Verfallserscheinungen und selbst die in den letzten Jahrzehnten außerhalb davon errichteten Einfamilienhäuser sind mittlerweile ebenfalls dem Verfall preisgegeben. Derzeit sind - abgesehen von den Gemeindehauptorten - kaum Bau- und Renovierungstätigkeiten auszumachen und neue Bewohner fallen ihrer Zahl und Auswirkung nach nicht ins Gewicht.

Das Gebiet nördlich der Typregion IIIa (IVb) weist ebenfalls kaum Newcomers auf, Siedlungen und Fluren sind aber in gepflegterem Zustand. Dies hängt damit zusammen, dass hier die abgewanderte einheimische Bevölkerung die Ortschaften an Wochenenden, gerade in der warmen Jahreszeit, aufsucht und dabei die alten Häuser renoviert oder als Freizeitdomizile im Hinblick auf eine künftige Dauerbesiedlung konserviert.

\section{Die demographischen Problemräume im alpinen Osten Österreichs}

Abbildung 1 zeigt, dass der alpine Osten Österreichs in der Zeit zwischen dem Zweiten Weltkrieg und 1981 insbesondere im Vergleich mit dem östlichen Abschnitt der italienischen Alpen und Teilen des alpinen Sloweniens kein ausgesprochenes Entvölkerungsgebiet war. Bis zur Gegenwart hat sich die Situation jedoch grundlegend verändert: Sieht man einmal vom (suburban geprägten) Wienerwald und vom Kärntner Zentralraum ab, so bildet dieser Alpenteil heute ein geschlossenes demographisches Problemgebiet, in dem nur ein paar Gemeinden einen positiven Wanderungssaldo aufweisen (vgl. Abb. 6).

Im Folgenden stellt sich somit die Frage nach dem Grund für diese besondere Entwicklung. Ferner wird zu erörtern sein, ob in diesem Abwanderungsraum das Phänomen der Amenity Migration - wie an den Beispielen von Friaul und Westslowenien aufgezeigt - bloß zeitlich verzögert einsetzen wird. Desgleichen ist zu klären, inwiefern solche Prozesse durch die spezifischen natur- und kulturräumlichen sowie wirtschaftspolitischen Bedingungen im Osten des alpinen Österreichs überhaupt möglich sind. 


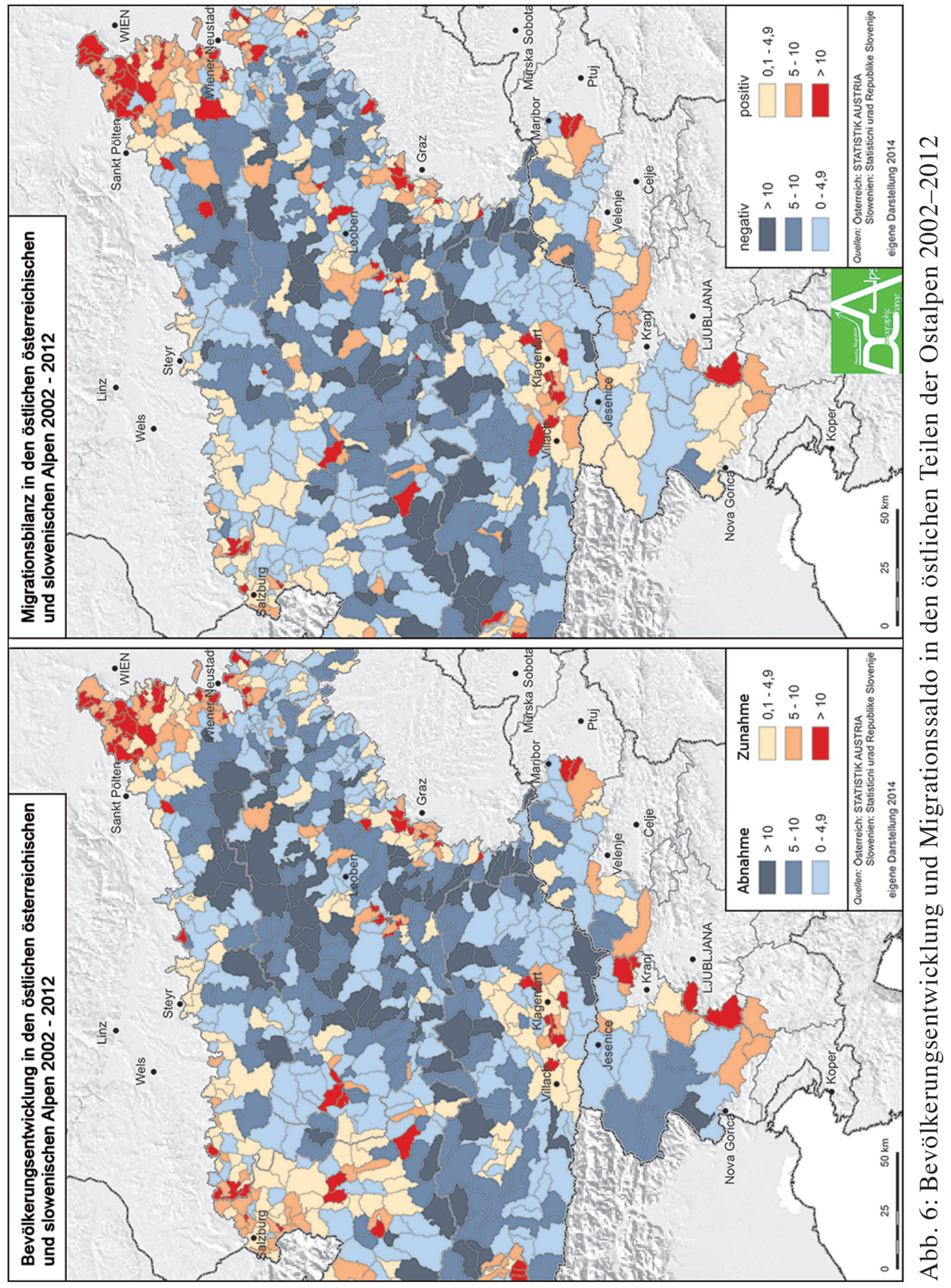




\subsection{Die Entwicklung zum Abwanderungsraum}

Die Ursachen, warum der Osten der österreichischen Alpen ein demographisches Problemgebiet geworden ist, lassen sich bis in die Zeit vor der Industrialisierung zurückverfolgen. So waren dort aufgrund der rechtsgeschichtlichen Benachteiligung (Freistiftrecht statt Erbbaurecht) bereits vor der Grundentlastung die Rahmenbedingungen für Innovationsprozesse bei weitem ungünstiger als in anderen alpinen Räumen. Dazu kamen in der Gründerzeit nichtbäuerliche urbane Waldinteressenten (Forsthuben statt Zuhuben - Lichtenberger 2002, S. 165-168; PenZ 2011, S. 48-49), die den regional bereits relevanten Großgrundbesitz maßgeblich erweiterten, wodurch die Persistenz rentenkapitalistischer Strukturen mit ihren Auswirkungen bis in die Gegenwart verstärkt wurde.

Ab den 1970er Jahren waren die Krise der alten Bergbau- und Industriegebiete (Region Eisenerz, Mur-Mürz-Furche, Hüttenberg), die verspätet einsetzende Tertiärisierung sowie der gegenüber dem westlichen Abschnitt der österreichischen Alpen, weiten Teilen der Schweiz und Südtirols unbedeutende Sommer- und Wintertourismus weitere Gründe dafür, dass sich der Osten zum Problemgebiet und in weiterer Folge zum Abwanderungsraum entwickelte. Als weiterer Faktor ist insbesondere in den kristallinen Mittelgebirgen die mit einer zeitlichen Verzögerung von bis zu zwei Jahrzehnten beginnende Deagrarisierung hervorzuheben. So verzeichneten noch die Ergebnisse der Volkszählung 1981 (ÖSTZA 1985) im Berggebiet Kärntens und der Steiermark einen überdurchschnittlich hohen Anteil in der Landwirtschaft Beschäftigter, der mit der Dominanz im Haupterwerb bewirtschafteter bäuerlicher Mittelbetriebe (ÖSTZA 1982, 1983) zusammenhängt. Darüber hinaus erfolgte die Abwanderung aus den peripher liegenden Einzelsiedlungen vorerst in die Gemeindehauptorte und noch nicht in größerem Ausmaße in die Zentralräume, die erst ab den 1980er Jahren als Migrationsziel an Bedeutung gewannen.

\subsection{Aktuelle Wanderungsbewegungen}

Ein Blick auf die aktuelle Zuwanderung im östlichen Abschnitt der österreichischen Alpen zeigt, dass diese - mit Ausnahme urbaner, suburbaner sowie in Pendlerdistanz zu den Zentralräumen liegender Gemeinden - nach wie vor unbedeutend ist. So dominieren sowohl in den alpinen Peripherräumen Kärntens und der Steiermark als auch Ober- und Niederösterreichs Gemeinden mit negativer Wanderungsbilanz. Auffallend starke Wanderungsverluste verzeichnen dabei die verkehrsgeographisch benachteiligten Gemeinden der Gurktaler Alpen (Politische Bezirke Feldkirchen und Sankt Veit an der Glan; STÖCKL 2014, S. 37).

Die Ursachen dafür liegen vor allem in fehlenden örtlichen Arbeitsplätzen, großen Pendlerdistanzen aber auch in der oft unzureichenden kommunalen Siedlungs- und Wohnbaupolitik. Die damit einhergehenden rückläufigen oder stagnierenden Geburtenraten spiegeln sich deutlich in der Altersstruktur der von der negativen demographischen Entwicklung besonders betroffenen Gemeinden wider. Folge davon ist 
eine insbesondere in den ohnehin bereits stark entvölkerten Ortsteilen außerhalb der Gemeindehauptorte zunehmend überalterte Wohnbevölkerung und damit einhergehend auch leerstehender oder ungenutzter Baubestand, der allerdings kaum für Amenity Migration Verwendung findet.

\subsection{Ursachen der unbedeutenden Amenity Migration}

Aus einer Analyse der Ursachen für die im alpinen Osten Österreichs unbedeutende Zuwanderung ergaben sich mehrere, aus der spezifischen rechtshistorischen und sozio-ökonomischen Entwicklung resultierende Faktoren, die vielfach nicht einzeln raumwirksam in Erscheinung treten, sondern bei regional unterschiedlicher Gewichtung verschieden stark miteinander vernetzt sind.

\subsubsection{Geringe landschaftliche Attraktivität aufgrund der naturräumlichen Rahmenbedingungen}

Diese resultiert unter anderem aus der gegenüber dem westlichen Abschnitt der österreichischen Alpen deutlich geringeren Seehöhe, die insbesondere in den Gurktaler Alpen (Kärnten, Steiermark) sowie im Steirischen Randgebirge (Steiermark, Kärnten, Niederösterreich) nur selten über $2.000 \mathrm{~m}$ erreicht. Umfangreiche Aufforstungen (vgl. Kap. 4.3.3) als Folge der seit der Gründerzeit massiven Bevölkerungs- und Siedlungsregression verstärken zudem die Wahrnehmung als dicht bewaldete Mittelgebirge (Andrian-Werburg et al. 2008; Seger 2005a-c, 2011). Betroffen davon sind die engen Seitengräben sowie die ausgedehnten Schattseiten der Haupttäler, bei denen es sich aufgrund von topographischen Gegebenheiten um ungeeignete Standorte für eine neue Zuwanderung handelt. Im Unterschied zu den übrigen Ostalpen gibt es zudem nur wenige oberhalb der Waldgrenze liegende Hochalmen bei gleichzeitiger Dominanz von Niederalmen unter der Dauersiedlungsgrenze, die aus aufgelassenen land- und forstwirtschaftlichen Betrieben entstanden sind und deren landschaftliche Attraktivität gleichfalls als gering empfunden wird.

Wenig geeignet für eine Neuzuwanderung sind auch weite Teile der steirischniederösterreichischen Kalkalpen - aufgrund der ungünstigen Reliefverhältnisse mit großen Vertikaldistanzen und periglazial geprägten überaus steilen Lagen, nicht zuletzt aber auch wegen des dort vorherrschenden kirchlichen und weltlichen Großgrundbesitzes (vgl. Kap. 4.3.4).

\subsubsection{Peripher liegende Einzelsiedlungen in benachteiligter Verkehrslage}

Kennzeichnend für den konvexen Mittelgebirgscharakter sind zudem die daraus resultierenden Lagekriterien der - mit Ausnahme der Haupttäler und inneralpinen Becken - dominierenden Einzelsiedlungen, die auch im Fall begünstigter topographischer Standorte im Bereich der Sonnseiten oft sehr abgelegen und somit nur schwer 
erreichbar sind. Dies trifft vor allem für jene Berggebiete zu, in denen der Verfall der bergbäuerlichen Kulturlandschaft bereits weit fortgeschritten ist (ČEDE 1991, 1998; PENZ 2005a) und daher auch die Verkehrsinfrastruktur sowohl für den Individual- als auch für den öffentlichen Verkehr nicht den aktuellen Anforderungen entspricht.

\subsubsection{Monotonisierung und Vereinheitlichung der Kulturlandschaft durch Aufforstung landwirtschaftlicher Nutzflächen}

Die Zunahme der Waldflächen insbesondere in den kristallinen Mittelgebirgen im östlichen Abschnitt der österreichischen Alpen durch Aufforstungen landwirtschaftlicher Nutzflächen ist ein weiterer wesentlicher Faktor der geringen Attraktivität der Berggebiete für Amenity Migration. Dadurch wird die bisher kleinräumig strukturierte Kulturlandschaft immer einheitlicher und monotoner (Borsdorf \& BENdER 2007; PENZ 2005b; TASSER \& TAPPEINER 2008). Die Ursachen dieser Entwicklung, die seit den 1970er Jahren zunehmend von bäuerlicher Seite getragen wird, sind eng mit der bereits diskutierten Bevölkerungs- und Siedlungsregression im Berggebiet verbunden sowie mit aktuellen Problemen in der alpinen Landwirtschaft - auch vor dem Hintergrund der Berggebietspolitik der EU. Räumlich beschränkt sich die Zunahme der Waldflächen mit ihren Fichtenmonokulturen in den vergangenen Jahrzehnten nicht nur (wie in der Gründerzeit) auf topographisch benachteiligte Standorte, sondern umfasst immer mehr auch die zurzeit noch dichter besiedelten Gunsträume (ČEDE 2000, S. 164-165; 2011). Dadurch verlieren auch die von Selbstanflug und Aufforstungen betroffenen Sonnseiten für potenzielle New Highlanders an Attraktivität.

\subsubsection{Großgrundbesitz und großbäuerliche Besitzstrukturen}

Nachteilig für die Ausbreitung der Amenity Migration ist zudem die Persistenz des Großgrundbesitzes, der bereits während der hochmittelalterlichen Kolonisation sowie durch das Bauernlegen der Gründerzeit entstanden ist. In der bergbäuerlichen Kulturlandschaft fand vor allem die zweite Phase in der systematischen Aufforstung der landwirtschaftlichen Nutzflächen jener Bergbauernbetriebe ihren sichtbaren Niederschlag, die vom Großbürgertum in der zweiten Hälfte des 19. Jhs. und darüber hinaus als Kapitalanlage oder Jagddomäne erworben wurden. Die damit einhergehende Bedeutung intensiver Forstwirtschaft (Kahlschläge, Forststraßen) manifestiert sich regional insbesondere in der zudem durch schlechte Verkehrsanbindung benachteiligten nordöstlichen Obersteiermark (Mariazeller Land) sowie in den angrenzenden Berggebieten Niederösterreichs (Ötscherland) (,Vergessene ländliche Räume“ - GRAmm et al. 2008).

Hervorzuheben sind ferner die großbäuerlichen Besitzstrukturen in den kristallinen Mittelgebirgen, wo ab den 1970er Jahren verstärkt aufgeforstet wurde (vgl. Kap. 4.3.3). Kennzeichen dieser Besitzverhältnisse sind gerade beim nichtbäuerlichen Großgrundbesitz starke Eigentumskontinuitäten und somit nur geringer, für Amenity Migration aber notwendiger Immobilienverkauf. 


\subsubsection{Unbefriedigende funktionale Ausstattung der Gemeindehauptorte}

Diese steht in enger Verbindung mit der allgemeinen Bevölkerungsabnahme im östlichen Abschnitt der österreichischen Alpen. Daher ist in zahlreichen Gemeindehauptorten keine Nahversorgung mehr vorhanden, und die soziale und technische Infrastruktur erweist sich ebenfalls als unbefriedigend (BORSDORF 2005; MACHOLD \& TAMME 2005; ÖROK 2006). Besonders drastisch ist die rückläufige Funktionsausstattung in den von Gemeindezusammenlegungen betroffenen ehemaligen Gemeindehauptorten sowie in den im weitläufigen Einzelsiedlungsgebiet der Mittelgebirge eingestreuten Kirchweilern, die gegenwärtig - von vereinzelten Ausnahmen abgesehen - überhaupt keine funktionale Ausstattung mehr aufweisen und somit für Amenity-Migranten gleichfalls wenig attraktiv sind.

\subsubsection{Schlechtes Image ehemaliger sowie in die Krise geratener Bergbau- und Industriegebiete}

Besonders betroffen davon sind im östlichen Abschnitt der österreichischen Alpen die Region um den Steirischen Erzberg sowie die Berggebiete beiderseits der in der Gründerzeit stark industrialisierten Mur-Mürz-Furche mit ihren in der zweiten Hälfte des 20. Jhs. geschlossenen oder mehrfach umstrukturierten alten Industriestandorten (BENDER 2011; Musil 2011, S. 20-21), die heute noch immer imagebestimmend sind. Beide Regionen konnten - trotz durchaus erfolgreicher Inwertsetzung ihres historischen Erbes (z.B. Steirische Eisenstraße), touristischer Förderungsmaßnahmen und anderer EU-kofinanzierter Regionalprojekte - ihr wenig positives Image bisher nicht entscheidend verbessern. Ähnliches gilt für die ober- und niederösterreichische Eisenwurzen (BRODA \& HeINTEL 2009) mit ihren ehemaligen eisenverarbeitenden Betrieben, wenngleich hier aufgrund der geringeren industriellen Überprägung der alpinen Kulturlandschaft bessere Voraussetzungen für Amenity Migration gegeben sind. Diese werden jedoch wiederum durch die periphere Lage der Region relativiert.

\section{Fazit}

Ausgehend vom statistischen Bild skizziert der vorliegende Beitrag zunächst die Trendwende im Migrationsgeschehen des Alpenbogens: Während der Westen eine Zuwanderungsregion geworden ist, scheint sich der Osten jenseits der Europaregion Tirol-Südtirol-Trentino zu einem ausgesprochenen Abwanderungs- und Entvölkerungsgebiet entwickelt zu haben. Qualitative Untersuchungen in diesen demographischen Problemräumen zeigen jedoch, dass dort zwar Fortzüge weiterhin vorherrschen, doch in den meisten Teilen genauso und gleichzeitig eine neue Zuwanderung feststellbar ist, die ähnliche Merkmale trägt wie in den Westalpen.

Die Beantwortung der Frage, ob sich im Osten dieselben Prozesse wie im Westen - nur zeitlich verzögert - abspielen, verlangt ein räumlich differenziertes Vorgehen. Generell lassen sich hierbei drei Raumkategorien unterscheiden: Die Montagna friula- 
na wurde von dem in diesem Beitrag mehrfach angesprochenen West-Ost-gerichteten demographischen Wandel bereits großteils erfasst. Wegen anhaltender berufs- und qualifikationsbedingter Abwanderung ergibt sich ein Nebeneinander von $\mathrm{Zu}$ - und Wegzügen, wobei ein negativer Migrationssaldo nach wie vor dominiert. Einwohnergewinne werden sich aber selbst bei positiver Wanderungsbilanz nicht einstellen, da die bevölkerungsbiologischen Gegebenheiten äußerst ungünstig sind. Diese Raumkategorie ist mittlerweile auch jenseits der italienisch-slowenischen Grenze ins Gemeindegebiet von Karfreit vorgerückt.

Ansonsten überwiegt im slowenischen Untersuchungsgebiet eine zweite Kategorie: Obwohl das naturräumliche Potenzial deutlich günstiger als im benachbarten Nordfriaul ist, haben New Highlanders diesen Raum bisher kaum entdeckt. Im Vergleich zu den westlich gelegenen Regionen spricht hier jedoch Vieles für eine bloße zeitliche Verzögerung des Eintreffens von Amenity-Migranten.

Als völlig anders erscheinen die gegenwärtigen demographischen Prozesse im alpinen Osten Österreichs, der inzwischen zum größten Problemgebiet des Alpenbogens geworden ist. Abbildung 7 fasst jene miteinander verknüpften Merkmale zusammen, welche diese dritte Raumkategorie von den beiden übrigen markant unterscheiden. In

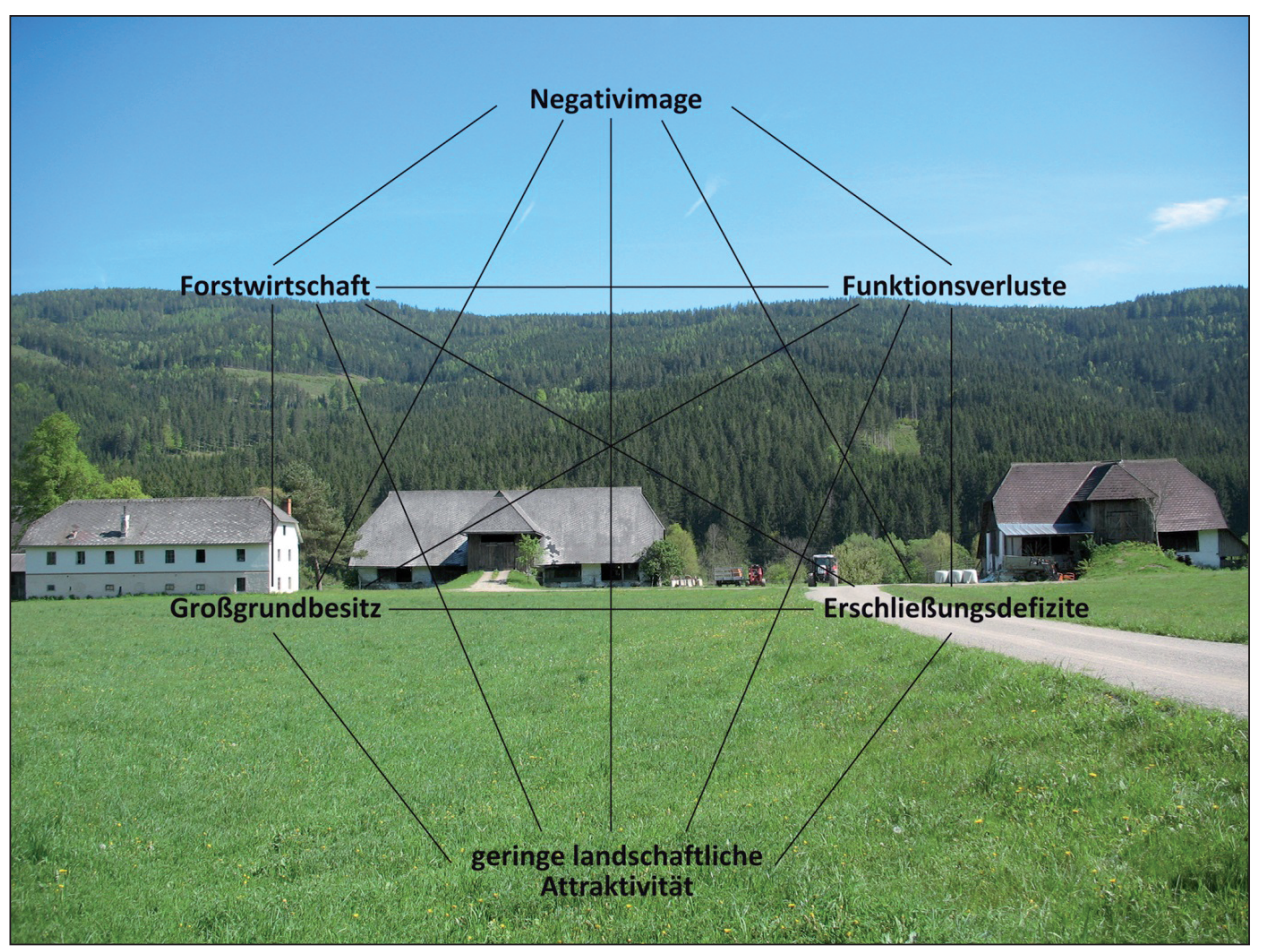

Abb. 7: Verknüpfung der Ungunstfaktoren für eine neue Zuwanderung - Beispielgemeinde Weitensfeld, Ortsteil Hafendorf, Gurktaler Alpen (Aufnahme der Autoren 2014) 
Kapitel 4.3 wurde erklärt, warum der östliche Teil der österreichischen Alpen nicht das Potenzial hat, Amenity-Migranten oder neue Bergbewohner anzulocken. Daher kann hier auch nicht von einer zeitlichen Verzögerung in der demographischen Trendwende gesprochen werden. Es ist im Gegenteil anzunehmen, dass die Ansiedlung von New Highlanders insbesondere in den kristallinen Mittelgebirgen der Länder Kärnten und Steiermark sowie in den „,vergessenen ländlichen Räumen“ der Niederösterreichischsteirischen Kalkalpen auch in Zukunft unbedeutend bleiben wird.

\section{Literaturverzeichnis}

Abrams J.B., Gosnell H., Gill N., Klepeis P. (2012), Re-creating the Rural, Reconstructing Nature: An International Literature Review of the Environmental Implications of Amenity Migration. In: Conservation and Society, 10, 3, S. 270-284.

Andrian-Werburg S., Fischer S., Schönthaler K. (2008), Waldflächenanteil, Zones boisées, Aree boscate, Gozdna površina. In: Tappeiner U., Borsdorf A., Tasser E. (Hrsg.), Alpenatlas, Atlas des Alpes, Atlante delle Alpi, Atlas Alp, Mapping the Alps. Society Economy - Environment, S. 216-217. Heidelberg, Spektrum Verlag.

B ̈̈TZING W. (2002), Die Bevölkerungsentwicklung der Alpen 1871-2000. Sonderbeilage zum CIPRA-Info 65.

Beismann M., Löffler R., Walder J., Steinicke E. (2012), Neue demographische Prozesse und deren Konsequenzen in den italienischen Alpen. In: Tagungsband der internationalen Konferenz „Wem gehören die Alpen“, 22.-24. September 2011, Agordo (Belluno). Padova, Universität Padua.

Bender O. (2011), Die vorindustrielle Metallverarbeitung am Beispiel der Eisenwurzen. In: Dittrich D., Jordan P., Musil R., Rumpolt P.A. (Hrsg.), Alpen - Lebensraum im Wandel. Die österreichischen Alpen im Blickpunkt der Geographie, S. 28-29. Wien, Österreichische Geographische Gesellschaft.

Bender O., Kanitscheider S. (2012), New immigration into the European Alps: emerging research issues. In: Mountain Research and Development, 32, 2, S. 235-241. - http:// dx.doi.org/10.1659/MRD-JOURNAL-D-12-00030.1 (zuletzt abgerufen im April 2014)

Bender O., Kanitscheider S. (2013), Amenity Migration in the Southern Andes and the Southern European Alps - A Key Factor for Sustainable Regional Development? In: Mitteilungen der Österreichischen Geographischen Gesellschaft, 155, S. 105-124.

Bender O., Pindur P. (2004), Erzberg, Eisenwurzen und „Mur-Mürz-Furche“. Entwicklung einer historischen Eisen- und Stahlindustrieregion in den österreichischen Alpen. In: Geographische Rundschau, 56, 5, S. 16-23.

Borsdorf A. (2005), Ohne „Greißler“ nicht zukunftsfähig? Die Grundversorgung der Alpengemeinden. In: Borsdorf A. (Hrsg.), Das neue Bild Österreichs. Strukturen und Entwicklungen im Alpenraum und in den Vorländern, S. 120-121. Wien, Österreichische Akademie der Wissenschaften.

Borsdorf A., Bender O. (2007), Kulturlandschaftsverlust durch Verbuschung und Verwaldung im subalpinen und hochmontanen Höhenstockwerk. Die Folgen des klimatischen und sozio-ökonomischen Wandels. In: Innsbrucker Geographische Gesellschaft (Hrsg.), Alpine Kulturlandschaft im Wandel. Hugo Penz zum 65. Geburtstag, S. 7-28. Innsbruck.

Brodda Y., Heintel M. (2009), Regionalentwicklung im Bereich inneralpiner Eisenindustrieund Bergbauorte. Das Beispiel Eisenwurzen. In: Hitz H., Wohlschlägl H. (Hrsg.), Das 
östliche Österreich und benachbarte Regionen. Ein geographischer Exkursionsführer, S. 313-332. Wien - Köln - Weimar, Böhlau.

Bufon M. (2003), Cross-border cooperation in the Upper Adriatic. In: Anderson J., O'Dowd L., WiLson T.M. (Hrsg.), New borders for a changing Europe. Cross-border cooperation and governance, S. 177-198. London, Routledge/Curzon.

Camenisch M., Debarbieux B. (2011), Inter-communal migrations in Switzerland: a "mountain factor"? In: Revue de Géographie Alpine/Journal of Alpine Research, 99, 1. - http:// rga.revues.org/1368 (zuletzt abgerufen im Februar 2014)

ČEDE P. (1991), Die ländliche Siedlung in den Niederen Gurktaler Alpen. Kulturlandschaftsverfall im Einzelsiedlungsgebiet unter dem Einfluss des Siedlungsrückganges (= Archiv für vaterländische Geschichte und Topographie, 71). Klagenfurt, Verlag des Geschichtsvereins für Kärnten.

ČEDE P. (1998), Kulturlandschaftswandel im Mittelgebirge. Fallbeispiel Niedere Gurktaler Alpen. In: Österreich in Geschichte und Literatur mit Geographie, 42, 6, S. 379-390.

ČEDE P. (2000), Verwaldung als Gefahr für die alpine Kulturlandschaft. In: ÖKosoziales FoRUM Österreich - Wintertagung 2000 (Hrsg.), Der Bauer im Spannungsfeld zwischen Wettbewerbsdruck und Konsumentenwünschen, S. 161-170. Gumpenstein, Verlag der Bundesanstalt für alpenländische Landwirtschaft.

ČEDE P. (2011), Kulturlandschaftsverfall und Verwaldung in den Niederen Gurktaler Alpen. In: Dittrich D., Jordan P., Musil R., Rumpolt P.A. (Hrsg.), Alpen - Lebensraum im Wandel. Die österreichischen Alpen im Blickpunkt der Geographie, S. 120-121. Wien, Österreichische Geographische Gesellschaft.

Corrado F. (2010), Ri-abitare le Alpi. Nuovi abitanti e politiche di sviluppo. Genova, Eidon.

Corrado F., Dematteis G., Di Gioia A. (Hrsg.) (2014), Nuovi montanari. Abitare le Alpi nel XXI secolo. Milano, Terre Alte-Dislivelli, Franco Angeli.

Di Giora A. (2013), Development perspectives for the Alpine urban systems. Spatial analysis upon role of services/Prospettive di sviluppo per i sistemi urbani delle Alpi. Un'analisi spaziale sul ruolo dei servizi. In: Mountain Dossier, 1, S. 24-30.

Europäische Akademie Bozen (EURAC) (Hrsg.) (2004), Indikatorensystem und Konzept für einen Alpenszustandsbericht, Anhang III - Definition des Perimeters der Alpenkonvention. Bozen.

Gosar A. (2005), The Cross-border Bricklaying Concept in the Alpen-Adria Region. In: Tourism Analysis, 10, 1, S. 65-78.

Gosar A. (Hrsg.) (2009), Razvojne priložnosti obmejnih območij Slovenije [Development opportunities of Slovenian border regions]. Koper, Annales Publishing House.

Gosar A., Roblek I. (2001), Die Zukunft des ländlichen Raumes in Slowenien. In: Mitteilungen der Österreichischen Geographischen Gesellschaft, 143, S. 131-148.

Gosnell H., Abrams J. (2011), Amenity migration: diverse conceptualizations of drivers, socioeconomic dimensions, and emerging challenges. In: GeoJournal, 76, 4, S. 303-322.

Gramm D., TASSER E., TAPPeIner U. (2008), Regionen gleicher Entwicklung, Régions à évolution comparable, Regioni a sviluppo similare, Območja s podobnim razvojem. In: TAPPEINER U., Borsdorf A., TAsser E. (Hrsg.), Alpenatlas, Atlas des Alpes, Atlante delle Alpi, Atlas Alp, Mapping the Alps. Society - Economy - Environment, S. 272-273. Heidelberg, Spektrum Verlag.

Hornung D., Röthlisberger T. (2005), Die Bergregionen in der Schweiz. Eidgenössische Volkszählung 2000. Neuchâtel, Bundesamt für Statistik.

Istituto Statale di Statistica (ISTAT) (Hrsg.) (1955ff.), Censimento generale della popolazione 1951, 1961, 1971, 1981, 1991, 2001. Roma. 
Istituto Statale di Statistica (ISTAT) (Hrsg.) (1971ff.), Popolazione e movimento anagrafico dei comuni (Statistiche demografiche/Annuario demografico). Roma (ab 2000 verfügbar unter http://www.demo.istat.it).

LAMPIČ B., MraK I. (2012), Globalization and foreign amenity migrants: the case of foreign home owners in the Pomurska region in Slovenia. In: European Countryside, 4, 1, S. 45-56.

Lichtenberger E. (2002²), Österreich. Geographie, Geschichte, Wirtschaft, Politik. Darmstadt, Wissenschaftliche Buchgemeinschaft.

Löffler R., Beismann M., Walder J., Steinicke E. (2014, im Druck), Newcomers in the European Alps. A West-to-East Running Process. In: Revue de Géographie Alpine/Journal of Alpine Research.

Löffler R., Steinicke E. (2007), Amenity Migration in the U.S. Sierra Nevada. In: Geographical Review, 97, 1, S. 67-88.

Machold I., Tamme O. (2005), Versorgung gefährdet? Soziale und wirtschaftliche Infrastrukturentwicklung im ländlichen Raum (= Forschungsbericht, 53). Wien, Bundesanstalt für Bergbauernfragen.

MCINTYRe N. (2009), Rethinking amenity migration: integrating mobility, lifestyle and socialecological systems. In: Die Erde, 140, 3, S. 229-250.

Messerli P., Scheurer T., Veit H. (2011), Between Longing and Flight - Migratory processes in mountain areas, particularly in the European Alps. In: Revue de Géographie Alpine/ Journal of Alpine Research, 99, 1. - http://rga.revues.org/1336 (zuletzt abgerufen im März 2014)

Moss L.A.G. (Hrsg.) (2006), The Amenity Migrants. Seeking and Sustaining Mountains and their Cultures. Wallingford (UK) - Cambridge (USA), CAB International.

Moss L.A.G., Glorioso R., Krause A. (Hrsg.) (2009), Understanding and Managing Amenityled Migration in Mountain Regions (= Proceedings of the Mountain Culture at the Banff Centre conference held May 15-19, 2008). Banff, International Amenity Migration Centre (IAMC).

Musil R. (2011), Kontinuitäten und Brüche. Industrielle Entwicklungspfade im Alpenraum. In: Dittrich D., Jordan P., Musil R., Rumpolt P.A. (Hrsg.), Alpen - Lebensraum im Wandel. Die österreichischen Alpen im Blickpunkt der Geographie, S. 13-23. Wien, Österreichische Geographische Gesellschaft.

ÖsterReichisches Raumordnungskonferenz (ÖROK) (Hrsg.) (2006), Aufrechterhaltung der Funktionsfähigkeit ländlicher Räume. Dienstleistungen der Daseinsvorsorge und Regionale Governance (= Schriftenreihe, 171). Wien, Österreichische Raumordnungskonferenz.

Österreichisches Statistisches Zentralamt (ÖSTZA) (Hrsg.) (1982, 1983), Land- und forstwirtschaftliche Betriebszählung 1980. Hauptergebnisse Kärnten, Steiermark (= Beiträge zur österreichischen Statistik, 660/2, 660/6). Wien.

Österreichisches Statistisches Zentralamt (ÖSTZA) (Hrsg.) (1985), Volkszählung 1981. Hauptergebnisse II, Kärnten, Steiermark (= Beiträge zur österreichischen Statistik, 630/13, 630/17). Wien.

Pascolini M. (Hrsg.) (2008), Le Alpi che cambiano. Nuovi abitanti, nuove culture, nuovi paesaggi. Die Alpen im Wandel. Neue Bewohner, neue Kulturen, neue Landschaften. Akten der internationalen Konferenz „Rete Montagna“ Tolmezzo, 16-17 November 2006. Udine, FORUM.

Penz H. (2005a), Vormarsch der Wälder auf Kosten der Landwirtschaft. Typen und Entwicklung der Hauptnutzungsformen. In: Borsdorf A. (Hrsg.), Das neue Bild Österreichs. Strukturen und Entwicklungen im Alpenraum und in den Vorländern, S. 72-73. Wien, Österreichische Akademie der Wissenschaften. 
Penz H. (2005b), Die „Talfahrt“ des (Berg-)Bauerntums. Der Bedeutungsverlust der Land- und Forstwirtschaft. In: BorsDorf A. (Hrsg.), Das neue Bild Österreichs. Strukturen und Entwicklungen im Alpenraum und in den Vorländern, S. 76-77. Wien, Österreichische Akademie der Wissenschaften.

Penz H. (2011), Von der bergbäuerlichen Kulturlandschaft zum Freizeitraum. In: DitTrich D., Jordan P., Musil R., Rumpolt P.A. (Hrsg.), Alpen - Lebensraum im Wandel. Die österreichischen Alpen im Blickpunkt der Geographie, S. 47-55. Wien, Österreichische Geographische Gesellschaft.

Perlik M. (2006), The Specifics of Amenity Migration in the European Alps. In: Moss L.A.G. (Hrsg.), The Amenity Migrants. Seeking and Sustaining Mountains and their Cultures, S. 215-231. Wallingford (UK) - Cambridge (USA), CAB International.

Perlik M. (2008), Neue Hoffnung Amenity Migration: Lassen sich die Erfahrungen aus den Rocky Mountains auf die Alpen übertragen? Amenity Migration, una nuova speranza: come transporre le esperienze delle Montagne Rocciose nelle Alpi? In: PAscolini M. (Hrsg.), Le Alpi che cambiano. Nuovi abitanti, nuove culture, nuovi paesaggi. Die Alpen im Wandel. Neue Bewohner, neue Kulturen, neue Landschaften, S. 143-157. Udine, FORUM.

Perlik M. (2011), Alpine gentrification: The mountain village as a metropolitan neighbourhood. In: Revue de Géographie Alpine/Journal of Alpine Research, 99, 1. - http://rga.revues. org/1370 (zuletzt abgerufen im April 2014)

Ravbar M. (2004), The Network of Settlements and the Development of Urbanization in Slovenia at the Start of the $21^{\text {st }}$ century. In: Slovenia - a Geographical Overview, S. 101-104. Ljubljana, Association of Geographical Societies in Slovenia, ZRC Publishing.

Schuler M., Perlik M., Pasche N. (2004), Nicht-städtisch, rural oder peripher - wo steht der ländliche Raum heute? Bern, ARE, Bundesamt für Raumentwicklung.

SEgER M. (2005a), Das Relief als landschaftsprägender Faktor. Landbedeckung und Nutzungsformen. In: Borsdorf A. (Hrsg.), Das neue Bild Österreichs. Strukturen und Entwicklungen im Alpenraum und in den Vorländern, S. 60-61. Wien, Österreichische Akademie der Wissenschaften.

Seger M. (2005b), Die Landschaft der österreichischen Alpen. Bewaldete Hügel im Osten, Hochgebirgskulisse im Westen. In: Borsdorf A. (Hrsg.), Das neue Bild Österreichs. Strukturen und Entwicklungen im Alpenraum und in den Vorländern, S. 50-51. Wien, Österreichische Akademie der Wissenschaften.

SEgER M. (2005c), Waldland Österreich. Ein Mosaik aus Laub- und Nadelwäldern. In: Borsdorf A. (Hrsg.), Das neue Bild Österreichs. Strukturen und Entwicklungen im Alpenraum und in den Vorländern, S. 22-23. Wien, Österreichische Akademie der Wissenschaften.

SEgER M. (2011), Differenzierung des österreichischen Alpenraumes nach dominanten agrarischen Nutzungen. In: Dittrich D., Jordan P., Musil R., Rumpolt P.A. (Hrsg.), Alpen - Lebensraum im Wandel. Die österreichischen Alpen im Blickpunkt der Geographie, S. 60-61. Wien, Österreichische Geographische Gesellschaft.

Statistični uRad Republike Slovenije (Hrsg.) (2013), SI-STAT podatkovni portal [SI-STATDatenportal]. Ljubljana. - http://pxweb.stat.si/pxweb/dialog/statfile1.asp (zuletzt abgerufen im Oktober 2012)

Steinicke E., Cirasuolo L., Čede P. (2011), “Amenity Migration” e la nuova tendenza demografica nelle aree tradizionali di spopolamento in Italia. In: Bollettino della Società Geografica Italiana, 13, 3, S. 273-293.

Steinicke E., ČEde P., Löffler R. (2012), In-migration as a new process in demographic problem areas of the Alps. Ghost towns vs. amenity settlements in the alpine border area between Italy and Slovenia. In: Erdkunde, 66, 4, S. 329-344. 
Steinicke E., Löffler R. (2013), Newcomers in the Italian Alps - Effects on Autochthonous Linguistic Minorities. Migration: Global Development, New Frontiers. Interdisciplinary conference on migration jointly organised by the NORFACE Research Programme on Migration and the Centre for Research and Analysis of Migration (CReAM) at UCL. 10-13 April, 2013, University College London. - https://cream.conference-services. net/resources/952/3365/pdf/MGDNF2013_0202.pdf (zuletzt abgerufen im April 2014)

Steinicke E., Čede P., Löffler R., Jelen I. (2014), "Newcomers" nelle regioni periferiche delle alpi. Il caso dell'area di confine tra Italia e Slovenia nelle Alpe Giulie. In: Rivista Geografica Italiana, 121, 1, S.1-20.

Steinicke E., Beismann M., Walder J., Löffler R., Jelen I. (2014), "Rifugi etno-linguistici" e tendenze demografiche attuali nelle Alpi italiane. Il caso di Sauris (Zahre). In: Porcellana V., Dí́moz F. (Hrsg.), Minoranze in mutamento. Etnicità, lingue e processi demografici nelle valli alpine italiane, S. 205-228. Alessandria, Edizioni dell 'Orso.

Stewart S.I. (2002), Amenity Migration. In Trends 2000: Shaping the Future. In: Luft K., MacDonald S. (Hrsg.), $5^{\text {th }}$ Outdoor Recreation \& Tourism Trends Symposium, Lansing, MI, 17-20 September 2000, S. 369-378. East Lansing (MI), Department of Park, Recreation, and Tourism Resources, Michigan State University.

SтöcKL P. (2014), Aktuelle Wanderungsbewegungen in alpinen Peripherräumen Kärntens. Das Phänomen der Amenity Migration und Zweit- bzw. Ferienwohnsitze am Beispiel der Gurktaler Alpen. Graz, Universität Graz, Masterarbeit am Institut für Geographie und Raumforschung.

TAsser E., TAppeiner U. (2008), Kulturlandschaftsvielfalt, Diversité du territoire, Diversitá del paesaggio, Raznolikost kulturne pokrajine. In: Tappeiner U., Borsdorf A., Tasser E. (Hrsg.), Alpenatlas, Atlas des Alpes, Atlante delle Alpi, Atlas Alp, Mapping the Alps. Society - Economy - Environment, S. 248-249. Heidelberg, Spektrum Verlag.

W Alder J., LöFfler R., Steinicke E. (2008), Autochthone ethno-linguistische Minderheiten in den italienischen Alpen im Lichte des aktuellen demographischen Wandels. In: Europa Regional, 16, 4, S. 178-190.

W Altert F., SchläPfer F. (2010), Landscape amenities and local development: A review of migration, regional economic and hedonic pricing studies. In: Ecological Economics, 70, S. 141-152. - http://dx.doi.org/10.1016/j.ecolecon.2010.09.031 (zuletzt abgerufen im April 2014) 